\title{
Synthesis of Steered Flat-top Beam Pattern Using Evolutionary Algorithm
}

\author{
Debasis Mandal $^{1 *}$, Kalyan S. Kola ${ }^{2}$, Jyotirmay Tewary ${ }^{3}$, \\ Ved P. Roy ${ }^{4}$, and Anup K. Bhattacharjee ${ }^{5}$ \\ 1,2,3,4 Bengal College of Engineering and Technology, Durgapur, India \\ ${ }^{5}$ National Institute of Technology, Durgapur, India \\ *corresponding author, E-mail: deb. mandal22@gmail.com
}

\begin{abstract}
In this paper a pattern synthesis method based on Evolutionary Algorithm is presented. A Flat-top beam pattern has been generated from a concentric ring array of isotropic elements by finding out the optimum set of elements amplitudes and phases using Differential Evolution algorithm. The said pattern is generated in three predefined azimuth planes instate of a single $\varphi$ plane and also verified for a range of azimuth plane for the same optimum excitations. The main beam is steered to an elevation angle of $30^{\circ}$ with lower peak SLL and ripple. Dynamic range ratio (DRR) is also being improved by eliminating the weakly excited array elements, which simplify the design complexity of feed networks.
\end{abstract}

\section{Introduction}

The scanned shaped beam has a wide range of applications in radar and satellite based communications. These shaped beams faced the problem of high side lobe and ripple. The concentric circular ring array antenna (CCRA) is very efficient to steer the beam patterns, which enables to increase the capacity in directional communication systems. Various techniques have been reported in the literature as given below [3-8].

Azevedo proposed a technique to generate shaped beam patterns from a linear array antenna through the control of nonuniformly samples of the array factor, both in amplitude and phase based on Fast Fourier Transform (FFT) [3]. By minimizing the First Null Beam Width (FNBW) and peak side lobe level (SLL) the best beam directivity has been obtained by Wooh and Shi to determine the optimum transducer parameters [4] and also investigated that the inter-element spacing and the number of elements plays an important role in determining transducer performances. Mandal et al. generates shaped beams from concentric ring array antenna using discrete excitations in a range of predefined $\varphi$ planes with zero elevation angle [5]. Reyna et al. described the design procedure of steerable concentric rings array for low side lobe level using Genetic Algorithm [6]. A new steerable reflector which can electronically steer a reflected beam over $+/-40^{\circ}$ in two dimensions for both polarizations have been proposed and implemented [7] by Sievenpiper et al. A different approach proposed by Mandal et al. for generating shaped beams (Flat-top and $\operatorname{cosec}^{2}$ )

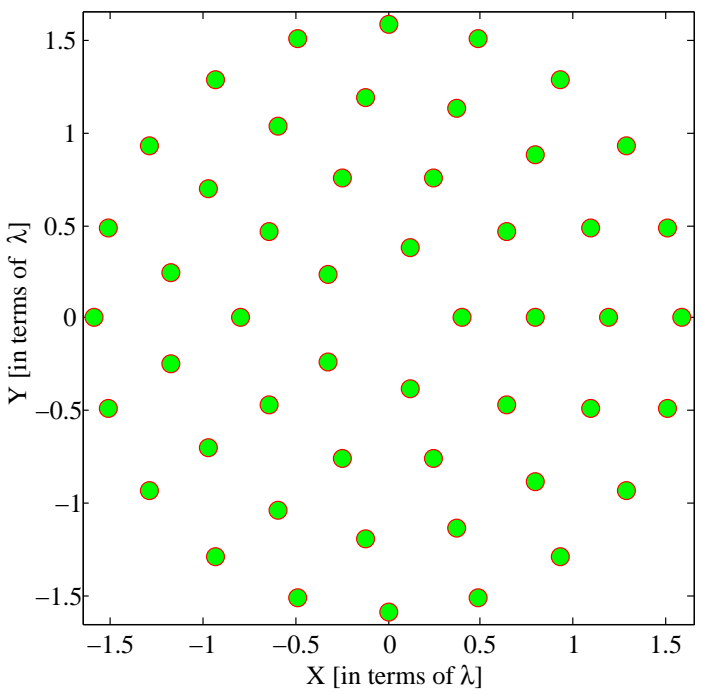

Figure 1: Geometry of concentric ring array Antenna

have been carried out using Differential Evolution Algorithm, which improves the dynamic range ratio by eliminating weekly excited array elements [8].

\section{Problem Formulation}

In this paper the peak SLL and ripple of the steered Flat-top beam pattern in three different planes of $\left(\theta_{0}=30^{\circ}, \varphi=\right.$ $\left.0^{\circ}\right),\left(\theta_{0}=30^{\circ}, \varphi=15^{\circ}\right)$ and $\left(\theta_{0}=30^{\circ}, \varphi=30^{\circ}\right)$ are reduced by finding out the optimum set of elements amplitudes and phases using Differential Evolution (DE) algorithm [9-12]. Scanning any beam from an array requires progressive phase gradient considered as $30^{\circ}$. Patterns are also generated in three arbitrarily chosen azimuth plane using same excitations, where the pattern retains their shape with some minor variations. Weakly excited array elements are removed (or turn off) from the optimized array without distorting the obtained pattern to achieve better Dynamic Range Ratio (DRR).

A concentric ring array of isotropic elements shown in Fig. 1 is considered. The far field pattern of the array scanned to a specified angle can be defined as $[1,2,6]$. 


$$
\begin{aligned}
& A F(\theta, \varphi)= \\
& \sum_{m=1}^{M} \sum_{n=1}^{N_{m}} I_{m n} e^{j k r_{m}\left[\sin \theta \cos \left(\varphi-\varphi_{m n}\right)-\sin \theta_{0} \cos \left(\varphi_{0}-\varphi_{m n}\right)\right]}
\end{aligned}
$$

where, $M=$ number of concentric rings;

$N_{m}=$ number of isotropic elements in $m$-th ring;

$I_{m n}$ excitation amplitude of $m n$-th element;

$r_{m}$ radius of the $m$-th ring;

$d_{m}$ inter element arc spacing of $m$-th circle;

$k=2 \pi / \lambda$, represents wave number;

$\lambda$ is the wave length;

$\theta, \varphi$ Elevation and azimuth angle;

$\varphi_{m n}=\frac{2 n \pi}{\lambda}$ is the angular location of the $m n$-th element with, $1 \leq \mathrm{n} \leq N_{m}$ and

$\left(\theta_{0}, \varphi_{0}\right)=$ steering angle.

Table 1: Ring radius and number of elements per ring

\begin{tabular}{lcccc}
\hline$M$ & 1 & 2 & 3 & 4 \\
\hline$N_{m}$ & 5 & 10 & 15 & 20 \\
$r_{m}(\lambda)$ & 0.3979 & 0.7958 & 1.1935 & 1.5915 \\
\hline
\end{tabular}

The fitness function of the Flat-top beam pattern for this approach is defined as:

$$
F(\rho)=k_{1}\left\{\text { peakSLL } L^{d}-\max _{\theta \in A}\left(A F_{d B}^{\rho}(\theta, \varphi)\right)\right\}^{2}+k_{2} \times \triangle
$$

where, $\triangle$ is defined for Flat-top beam pattern as:

$$
\triangle=\sum_{\theta_{i} \in\left\{-15^{\circ}+\theta_{\circ} \text { to } 15^{0}+\theta_{\mathrm{o}}\right\}}\left|A F_{d B}^{\rho}\left(\theta_{i}, \varphi\right)-D\left(\theta_{i}, \varphi\right)\right|
$$

In equation, 2 and $3 \varphi \in\left(0^{\circ}\right.$ to $\left.30^{\circ}\right)$ at $\theta=30^{\circ}$ plane.

$\rho$ is the unknown parameter set responsible for the desired beam patterns. Which is defined as follows:

$$
\rho=\left\{I_{m n}, \alpha_{m n}\right\} ; \quad 1 \leq m \leq M \text { and } 1 \leq n \leq N_{m}
$$

peakSLL $L^{d}$ are the desired value of peak SLL for Flattop beam pattern. $A$ is the sidelobe region for the pattern. $D_{d B}(\theta, \phi)$ is the desired pattern shown in Fig. 2 at $\varphi=$ $0^{\circ}, 15^{\circ}$ and $30^{\circ}$ plane. The range of $\theta_{i}$ for Flat-top beam pattern is $-15^{\circ}+\theta_{o}$ to $15^{\circ}+\theta_{\mathrm{o}}$.

$k_{1}$ and $k_{2}$ are the weighting factors. The fitness function has to be minimized by finding out optimum set of amplitudes and phases using Differential Evolution (DE) Algorithm.

\section{Differential Evolution Algorithm}

Differential Evolution Algorithm (DE) is one of the best population based evolutionary Algorithm introduced by Storn and Price in the year of 2005, which is able to find out

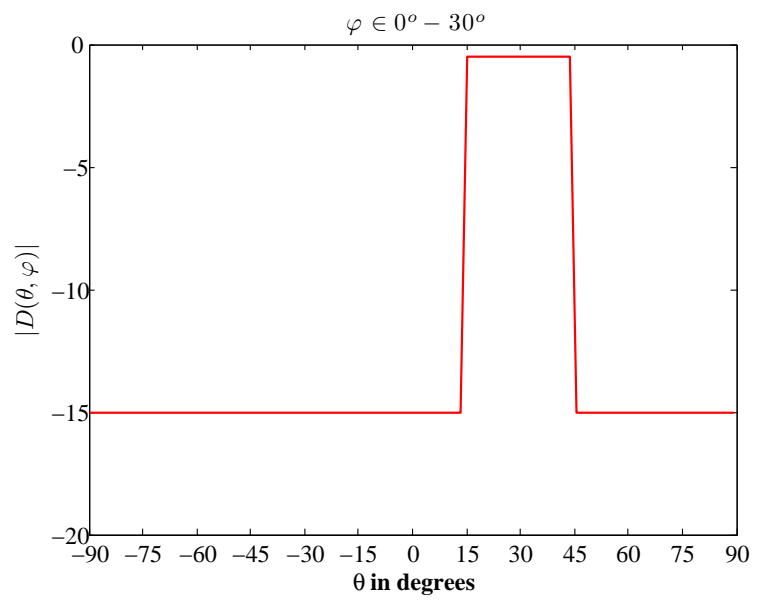

Figure 2: Desired scanned Flat-top beam pattern

true global minima with less control parameters and faster convergence. After a randomly initialization and generation in $D$ dimensional search space three main steps involved in DE Algorithm are [8-12] namely Mutation, Crossover and Selection. In Mutation process mutant vector $V_{i, G}$ is generated corresponding to the target vector $X_{i, G}$. During crossover operation the trial vector $U_{i, G}$ is generated using mutant vector $V_{i, G}$ and target vector $X_{i, G}$. Finally, in Selection process the objective function values are compared with each trial vector and those produces the best fitness function retain for the next generation. These steps are being repeated till the predefined value of generation, results the best solution of the objective function in the current population $\left(X_{\text {best }, G}\right)$.

Pseudo code of Differential Evolution Algorithm (DE)

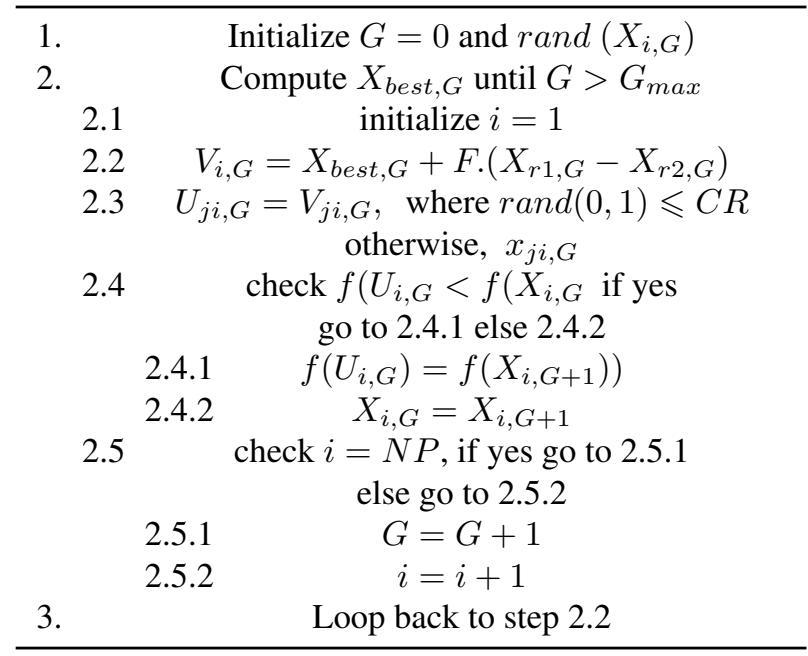

The population size, scale factor $(F)$, and crossover rate $(C R)$ of $\mathrm{DE}$ are chosen as $50,0.8$, and 0.2 respectively [8-12],. The DE scheme used in this paper is " $D E /$ best/1/bin" with maximum iteration number of 5000 . 

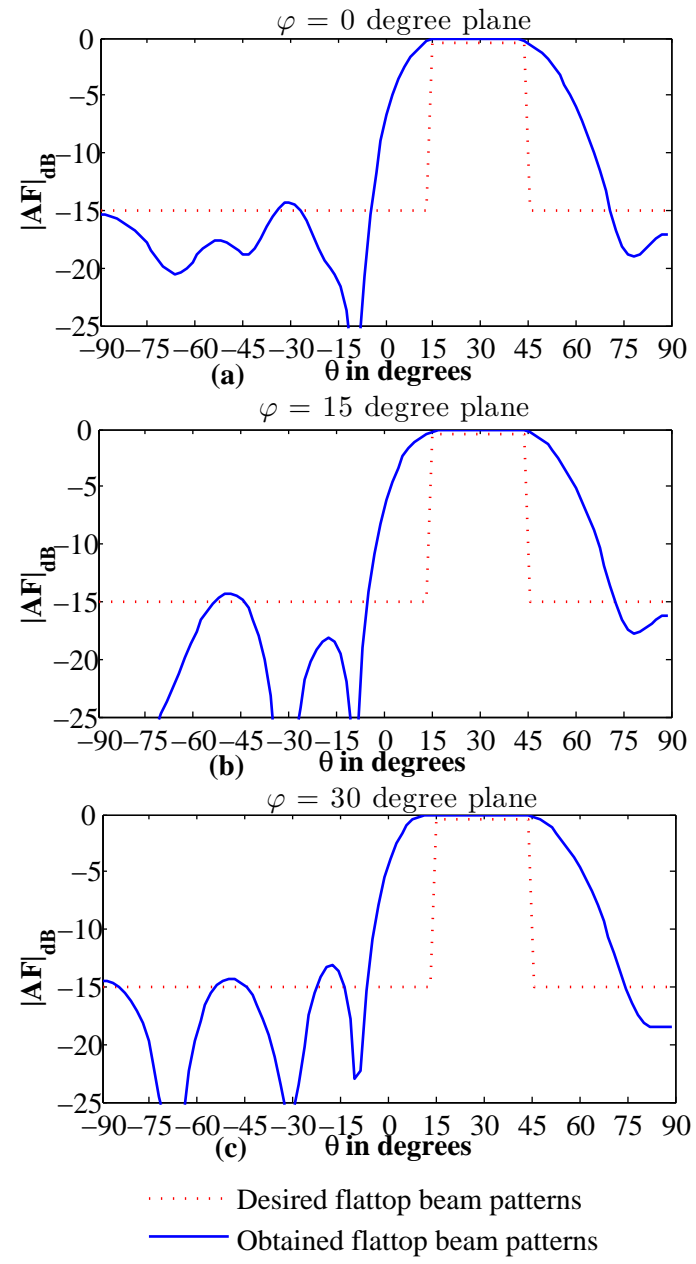

Figure 3: Scanned Flat-top patterns after removing the weakly excited elements from the array (a) $\varphi=0^{\circ}$ (b) $\varphi=15^{\circ}$ (c) $\varphi=30^{\circ}$

\section{Simulation Results}

A concentric ring array of 50 isotropic elements with $0.5 \lambda$ inter-element spacing has been considered (Figure 1).

Table 2: Desired and obtained result for scanned Flat-top pattern in predefined $\varphi$ planes

\begin{tabular}{|c|c|c|c|c|}
\hline $\begin{array}{c}\theta_{o} \\
\text { (Degree) }\end{array}$ & $\begin{array}{c}\varphi \\
\text { (Degree) }\end{array}$ & $\begin{array}{c}\text { Design } \\
\text { Parameters }\end{array}$ & $\begin{array}{l}\text { Flat-top } \\
\text { Desired }\end{array}$ & $\begin{array}{l}\text { Pattern } \\
\text { Obtained }\end{array}$ \\
\hline \multirow{3}{*}{$30^{\circ}$} & $\varphi=0^{\circ}$ & $\begin{array}{c}\text { Peak SLL }(\mathrm{dB}) \\
\triangle(\mathrm{dB})\end{array}$ & $\begin{array}{c}-15.00 \\
0.00\end{array}$ & $\begin{array}{c}-14.2675 \\
9.8496\end{array}$ \\
\hline & $\varphi=15^{\circ}$ & $\begin{array}{c}\text { Peak SLL }(\mathrm{dB}) \\
\triangle(\mathrm{dB})\end{array}$ & $\begin{array}{c}-15.00 \\
0.00\end{array}$ & $\begin{array}{c}-13.5942 \\
9.5019\end{array}$ \\
\hline & $\varphi=30^{\circ}$ & $\begin{array}{c}\text { Peak SLL }(\mathrm{dB}) \\
\triangle(\mathrm{dB})\end{array}$ & $\begin{array}{c}-15.00 \\
0.00\end{array}$ & $\begin{array}{l}-13.15 \\
9.8783\end{array}$ \\
\hline
\end{tabular}

The number of elements present in each ring with their respective ring radius is given in Table 1 . Figure 2 and Figure 3 depict the desired and obtained Flat-top beam pattern in all predefined azimuth planes considering the scanning angle of $30^{\circ}$ respectively.

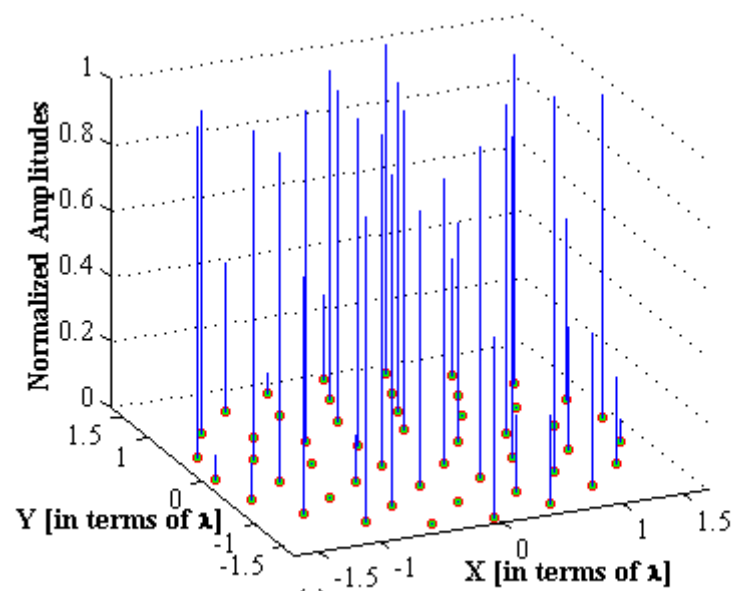

(a)

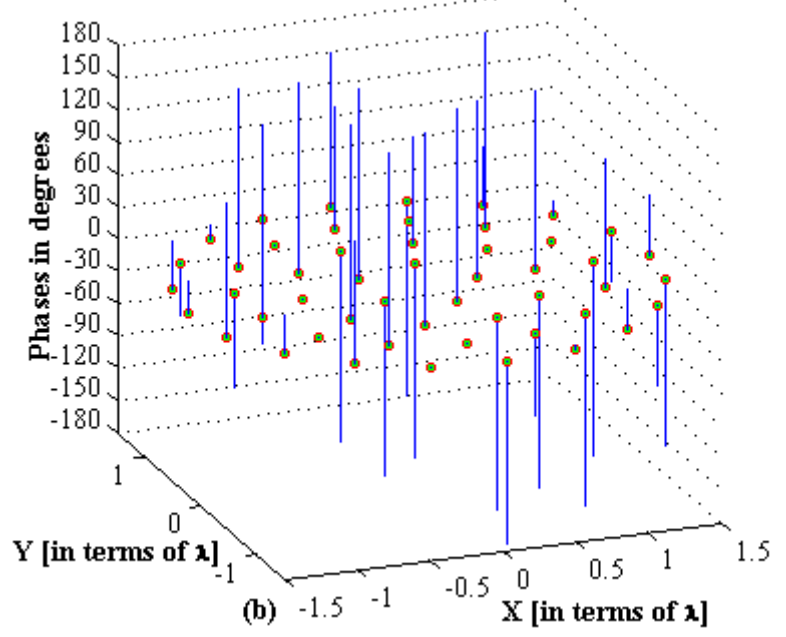

Figure 4: Optimum excitations (a) Amplitudes and (b) Phases

The design specification of steered Flat-top beam pattern with its corresponding desire and obtained results are given in Table 2. From Table 2 it can be observed clearly that the peak SLL for all three predefined azimuth planes are very much closer to its desired value.

Optimum values of excitation for the array elements obtained using the DE algorithm are presented in Table 3 where the elements having amplitudes less than 0.04 considered as 'of $f^{\prime}$, by making impedance equal to the source impedance of the fed elements resulting improvement in DRR level. The obtained values of normalized amplitudes having a range in between 0.0125 to 1 provide the DRR level 80. Whereas, by making the elements 'of $f^{\prime}$ having amplitudes less than 0.04 , the range of amplitudes are now become 0.059 to 1 results improved DRR level 16.949 without distorting the shape of the beam. Thus simplify the de- 
Table 3: Amplitudes and Phases of the Array Elements

\begin{tabular}{|c|c|c|c|c|}
\hline $\begin{array}{l}\text { Ring } \\
\text { No. }\end{array}$ & $\begin{array}{l}\text { Normalized } \\
\text { Amplitudes }\end{array}$ & \multicolumn{3}{|c|}{$\begin{array}{c}\text { Phases } \\
\text { in Degrees }\end{array}$} \\
\hline 1. & $\begin{array}{l}0.603,0.969,0.992, \\
1.000,0.860,\end{array}$ & $\begin{array}{l}162.72 \\
-161.64\end{array}$ & $\begin{array}{l}-180.00 \\
178.20\end{array}$ & 177.12, \\
\hline 2. & $\begin{array}{l}1.000, \quad 0, \quad 1.000, \\
1.000,1.000, \quad 0, \\
0.141,0.834,1.000, \\
0.970,\end{array}$ & $\begin{array}{c}166.68 \\
-176.04 \\
180.00 \\
-180.00\end{array}$ & $\begin{array}{c}0 \\
177.48 \\
180.00\end{array}$ & $\begin{array}{c}99.36 \\
0, \\
-180.00\end{array}$ \\
\hline 3. & $\begin{array}{ccc}1.000, & 0, & 0.524, \\
0, & 1.000, & 0, \\
0.321, & 1.000, & 0.997, \\
0, & 1.000, & 0, \\
0.229, & 0.629, & 0.371,\end{array}$ & $\begin{array}{c}-180.00 \\
0 \\
167.40 \\
0 \\
-76.32\end{array}$ & $\begin{array}{c}0, \\
113.76 \\
-87.84 \\
180.00 \\
-180.00\end{array}$ & $\begin{array}{c}179.28 \\
0, \\
180.00 \\
0 \\
119.16\end{array}$ \\
\hline 4. & $\begin{array}{ccc}0.985 & 0.544 & 0.999 \\
0.354 & 0.999 & 0.258 \\
0.059 & 0.452 & 0.982 \\
1.000 & 0.075 & 0.107 \\
0.720 & 0.930 & 0 \\
0.549 & 0.271 & 0.467 \\
0.262 & 0.063 & \end{array}$ & $\begin{array}{c}54.36 \\
54.00 \\
-115.56 \\
45.00 \\
35.64 \\
-168.84 \\
-73.44\end{array}$ & $\begin{array}{c}-45.00 \\
-180.00 \\
12.96 \\
31.32 \\
114.84 \\
3.24 \\
-154.08\end{array}$ & $\begin{array}{c}12.60 \\
142.56 \\
-48.60 \\
123.84 \\
0 \\
37.80\end{array}$ \\
\hline
\end{tabular}

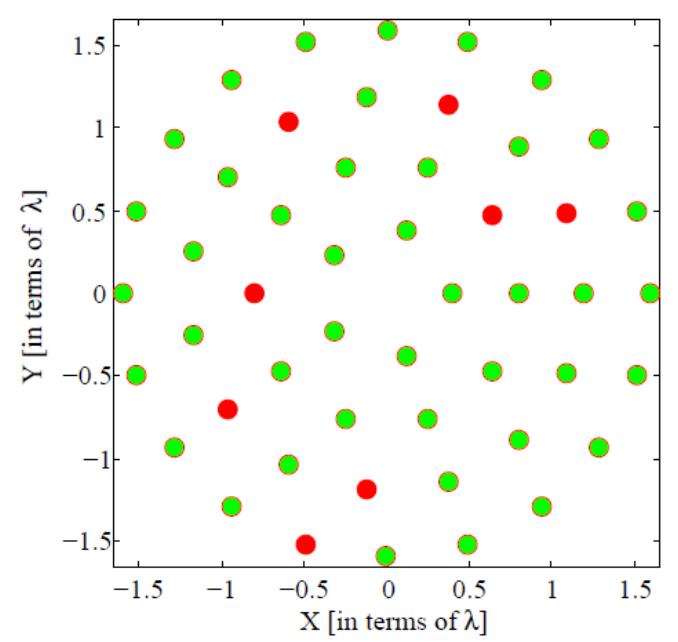

Figure 5: Optimized array after removing weekly excited elements (green represents “on” and red “off”)

sign complexity of feed networks (less number of attenuators and phase shifters are required). Figure 4(a) and Figure 4(b) depicts element wise normalized amplitudes and phases respectively.

After eliminating weakly excited array elements the modified array geometry is shown in Figure 5. The excitations are also being verified by considering three arbitrarily chosen azimuth planes $\left(\varphi=7.5^{\circ}, 22.5^{\circ}\right.$ and $\left.35^{\circ}\right)$ presented in Figure 6 where the steered beam pattern has been generated with some minor variations with respect to its desired parameters illustrated in Table 4.
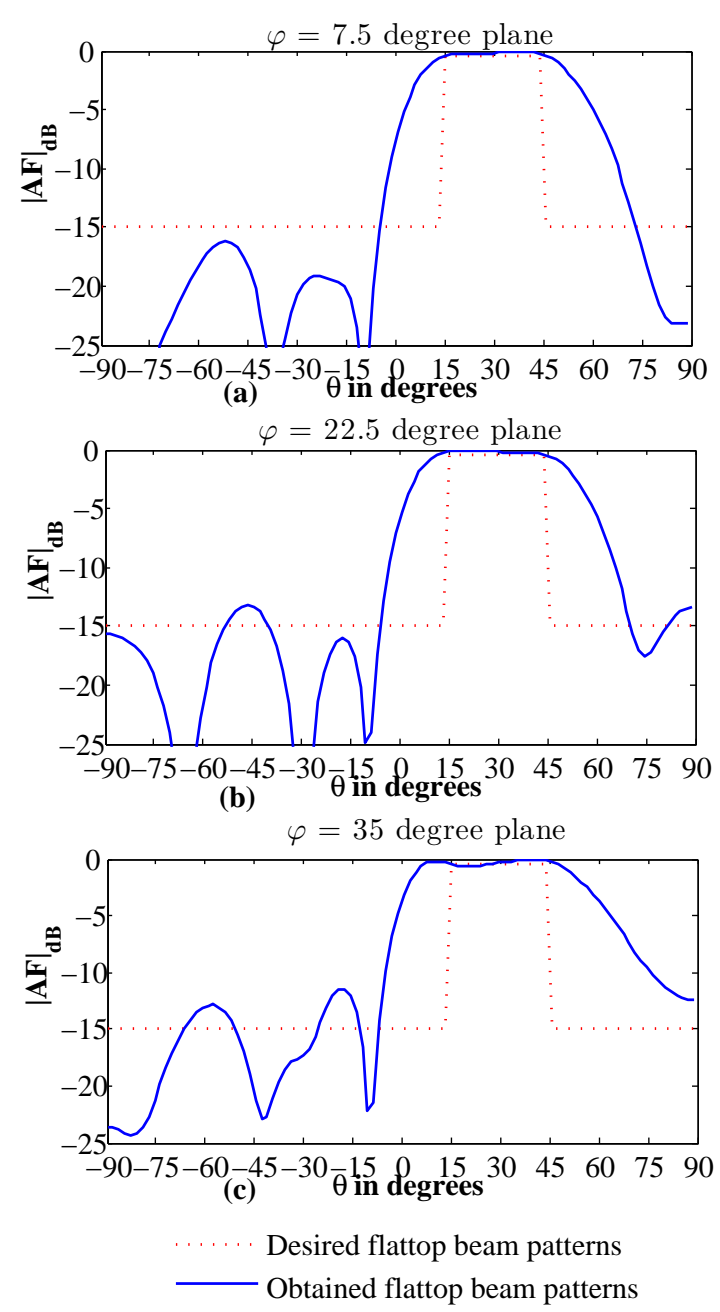

Figure 6: Scanned Flat-top patterns in arbitrary planes (a) $\varphi=7.5^{\circ}$ (b) $\varphi=22.5^{\circ}$ (c) $\varphi=35^{\circ}$.

Table 4: Obtained results for scanned Flat-top pattern in arbitrary $\varphi$ planes

\begin{tabular}{|c|c|c|c|}
\hline $\begin{array}{c}\theta_{o} \\
\text { (Degree) }\end{array}$ & $\begin{array}{c}\varphi \\
\text { (Degree) }\end{array}$ & $\begin{array}{l}\text { Design } F \\
\text { Parameters }\end{array}$ & $\begin{array}{c}\text { Flat-top Pattern } \\
\text { Obtained }\end{array}$ \\
\hline \multirow{3}{*}{$\theta_{o}=30^{\circ}$} & $\varphi=7.5^{\circ}$ & $\begin{array}{c}\text { Peak SLL }(\mathrm{dB}) \\
\triangle(\mathrm{dB})\end{array}$ & $\begin{array}{l}-16.2995 \\
11.3549\end{array}$ \\
\hline & $\varphi=22.5^{\circ}$ & $\begin{array}{l}\text { Peak SLL } \\
\triangle(\mathrm{dB})\end{array}$ & $\begin{array}{c}-13.2957 \\
10.8354\end{array}$ \\
\hline & $\varphi=35^{\circ}$ & $\begin{array}{l}\text { Peak SLL } \\
\triangle(\mathrm{dB})\end{array}$ & $\begin{array}{l}-11.46 \\
14.3837\end{array}$ \\
\hline
\end{tabular}


From Table 4 it can be observed that for an arbitrary angle of $7.5^{\circ}$ which is in-between the range of predefined plane $0^{\circ}$ to $15^{\circ}$ has a value of peak SLL $-16.2995 \mathrm{~dB}$ and ripple $(\triangle) 11.3549 \mathrm{~dB}$ where as for the arbitrary angle of $22.5^{\circ}$ the value of peak SLL and ripple $(\triangle)$ are -13.2957 $\mathrm{dB}$ and $10.8354 \mathrm{~dB}$ respectively which is adequate to its desired value but for an arbitrary angle of $35^{\circ}$ which is beyond the pre defined range of azimuth plane $\left(\varphi=30^{\circ}\right)$ the obtained peak SLL and ripple are far deviated from its desired value. The convergence curve of DE algorithm has been given in Figure 7.

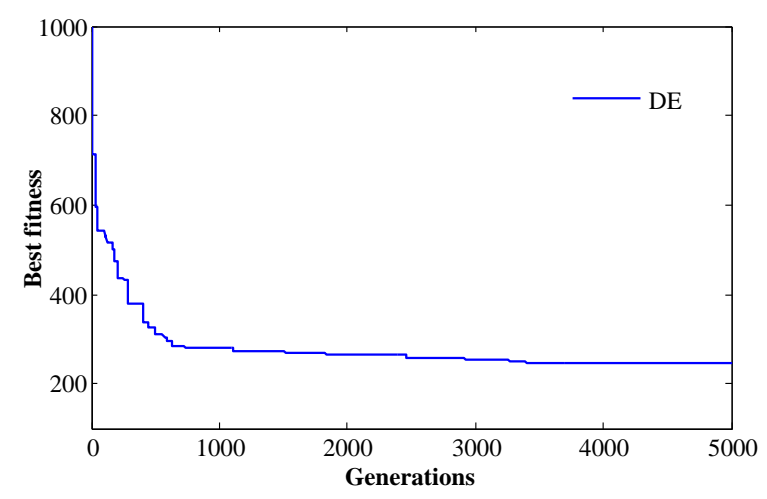

Figure 7: Convergence curve of Differential Evolution Algorithm (DE).

\section{Conclusions}

Synthesis of a Flat-top beam pattern of concentric ring array antenna for a scanning angle of $30^{\circ}$ has been proposed and assessed using Differential Evolution algorithm. This pattern has been generated in three predefined azimuth planes instead of any particular $\varphi$ plane. Optimum excitations used for generating the Flat-top beam pattern are also verified for three arbitrarily chosen azimuth angles to illustrate that those excitations are useful not only in some predefined $\varphi$ planes rather a range of azimuth plane. For synthesis of Flat-top beam patterns; side lobe and ripple are minimized by minimizing properly formulated fitness function using evolutionary Algorithm. Dynamic Range Ratio (DRR) have also been improved by turning off the weakly excited array elements. The presented method can also be applied to synthesize other array geometries.

\section{References}

[1] Balanis, C. A., Antenna Theory, Analysis and design, 3rd ed. New York, USA: Jhon Willy \& sons, 1997.
[2] Elliott, R. S., Antenna Theory \& Design, Revised Edition. Wiley-IEEE Press, Dec, 2002.

[3] Azevedo, J. A. R, Shaped beam pattern synthesis with nonuniform sample phases, Progress in Electromagnetics Research B, Vol. 05, 77-90, 2008.

[4] Wooh, S. C, and Shi, Y, Optimum beam steering of linear phased arrays, Wave Motion, Vol. 29,No. 03, 245-265, 1999.

[5] Mandal, D., Chatterjee, A. and Bhattacharjee, A. K. Design of Fully Digital Controlled Shaped Beam Synthesis Using Differential Evolution Algorithm, International Journal of Antennas and Propagation, Vol. 2013, 1-9, 2013.

[6] Reyna, A., Panduro, M. A., Covarrubias, D. H., and Mendez, A. Design of steerable concentric rings array for low side lobe level, Scientia Iranica, Vol. 19, No. 03, 727-732, 2012.

[7] Sievenpiper, D. F., Schaffner, J. H, Song, H. J., Loo, R. Y. and Tangonan, G. Two-dimensional beam steering using an electrically tunable impedance surface, IEEE Transactions on Antennas and Propagation, Vol. 51, No. 10, 2713-2722, 2003.

[8] Mandal, D., Chatterjee, A., Mondal, T. and Bhattacharjee, A. K. Shaped beam synthesis of concentric ring array antenna using Differential Evolution Algorithm, International Journal of Electronics and Communications (AEU), Vol. 69, No. 07, 1025-1032, 2015.

[9] Storn, R., Price, K., Differential evolution: a simple and efficient heuristic for global optimization over continuous spaces, Journal of Global Optimization, Vol. 11, No. 04, 341-359, 1997.

[10] Price, K. V., Storn, R. M. and Lampinen, J. A., Differential EvolutionA Practical Approach to Global Optimization. Natural Computing,Springer, New York, USA, 2005.

[11] Das, S., Abraham, A., Chakraborty, U. K., and Konar, A., Differential evolution using a neighborhood-based mutation operator, IEEE Transactions on Evolutionary Computation, Vol. 13, No. 03, 526-553, 2009.

[12] Guo, J., Li, J., Pattern synthesis of conformal array antenna in the presence of platformusing differential evolution algorithm, IEEE Transactions on Antennas and Propagation, Vol. 57, No. 09, 2615-2621, 2009. 